\title{
ENERGY CHANGES IN IDEAL FIBER-REINFORCED COMPOSITES*
}

\author{
$\mathrm{BY}$ \\ A. C. PIPKIN \\ Brown University
}

1. Introduction. In the present paper we consider a problem of finite plane deformation of an ideal fiber-reinforced material [1, 2]. A slab of material (Fig. 1) is deformed by a load applied to its end. The problem has more than one statically and kinematically admissible solution. Two of these are shown in Figs. 2 and 3.

In Sec. 3 we show that the deformation in Fig. 2 satisfies the minimum-energy criterion, while that in Fig. 3 does not. In fact, the energy is not even stationary at equilibrium for the deformation in Fig. 3. Minimum energy requires satisfaction of the stress equations of equilibrium, but the converse is not true. In Sec. 5 we show that the non-stationary energy at equilibrium is caused by the discontinuity line AD in Fig. 3. Thus, solutions with discontinuity lines are energetically inadmissible, and uniqueness is restored by using the minimum-energy principle.

In Sec. 6 we turn to a different matter, which also involves consideration of the energy. We compute the energy dissipated when the slab separates from the support at point $A$, along a small segment of the boundary. We show that the energy dissipation does not take place at the crack tip $A$, but at the point $D$ on the opposite boundary. We speculate on the possible implication of this result for fracture analysis.

2. The problem and two of its solutions. Fig. 1 shows a slab of ideal fiber-reinforced material [1,2] bonded to a rigid support along one of its ends and part of its lower boundary. The slab is composed of inextensible fibers that are parallel to the $X$-direction when the slab is not deformed, and the material is incompressible in bulk. The shearing stress response of the material is elastic.

The slab is finitely deformed by applying a line load $F$, per unit of length normal to the plane of the diagram, to the unsupported end. We assume plane strain conditions. Except where the line load is applied and where the slab is bonded to the rigid support, the surface of the slab is free from traction.

Figs. 2 and 3 show two deformations that satisfy all of the kinematic constraint conditions and static equilibrium conditions of the problem. The deformation in Fig. 2 is essentially the same as in some previously-solved cantilever and column-buckling problems $[2,3]$, and it is the only solution that involves no deformation of the material in the region $X<0$. The same kind of deformation has been assumed to be valid in a machining problem [4] and in a problem involving the inflation of a central crack [5]; in the latter problem the deformation is different in detail but is similar to the present one in that it is assumed that there is no deformation in the region $X<0$. Deformations of the type shown

\footnotetext{
* Received July 29, 1977. The work described in this paper was carried out under grant MCS76-08808 from the National Science Foundation. The author gratefully acknowledges this support.
} 


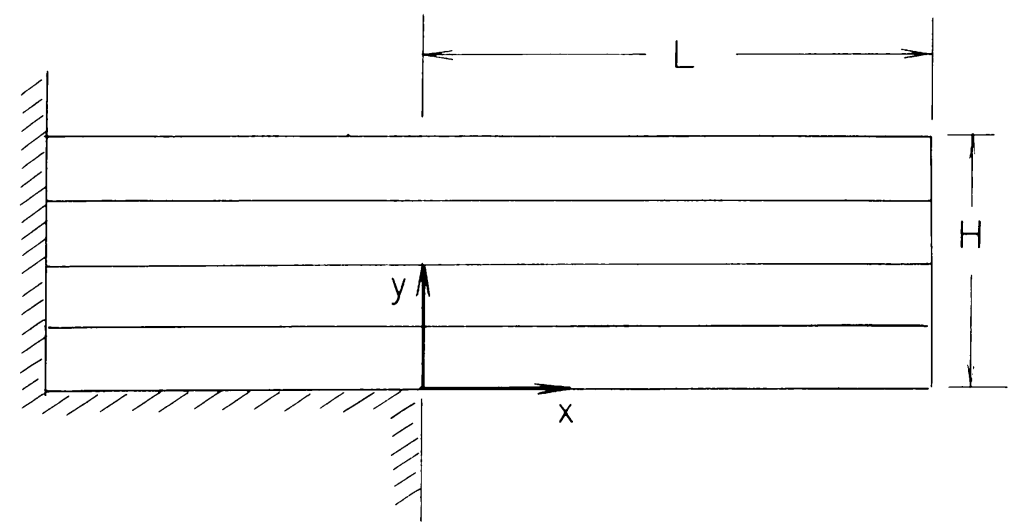

Fic;. 1. Undeformed slab.

in Fig. 3 are statically and kinematically admissible in these problems [4, 5], and the reason for rejecting such a solution has not been explained previously. In the present paper we show that the deformation in Fig. 3 is energetically inadmissible.

The deformations in Figs. 2 and 3 are kinematically admissible because every segment of every fiber has the same length in the deformed body that it had when the body was undeformed, and volume is then preserved because the spacing between fibers is the same as it was initially. Preservation of fiber spacing requires the crimp line $A D$ in Fig. 3 to bisect the angle between the fiber directions on either side of it.

Let $\theta(x, y)$ be the angle that the fiber through the point $x, y$ makes with the horizontal direction. Since the fiber was initially horizontal, this is the deflection angle of the fiber. Let $k(x, y)$ be the amount of shear at the point $x, y$. This is the parallel relative displacement of two neighboring fibers, divided by the distance between them. It is a

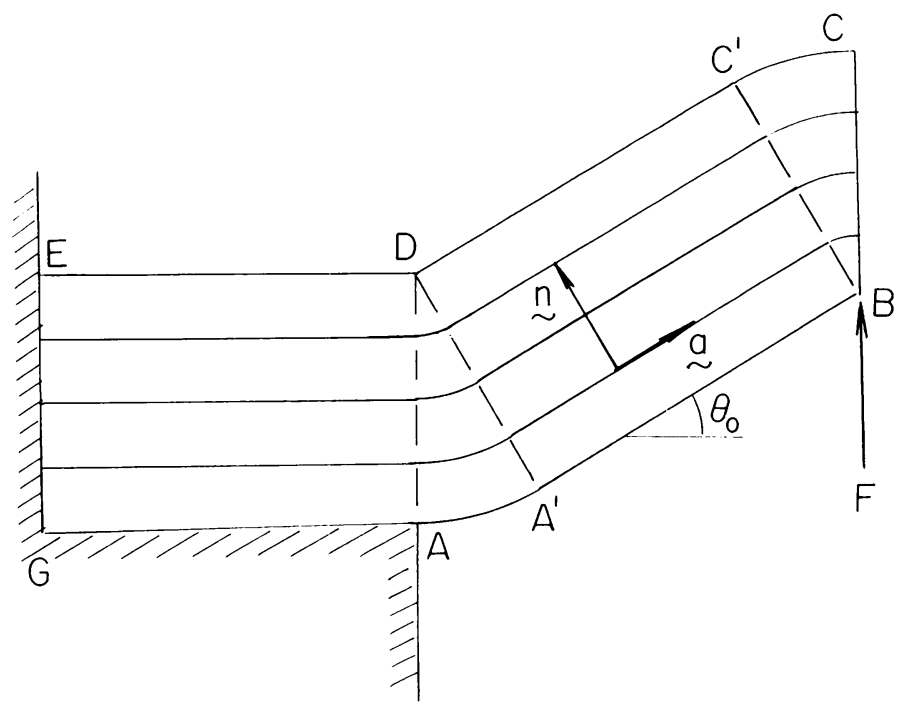

FIG. 2. Deformation with smooth change of fiber direction in region $D A A^{\prime}$. 


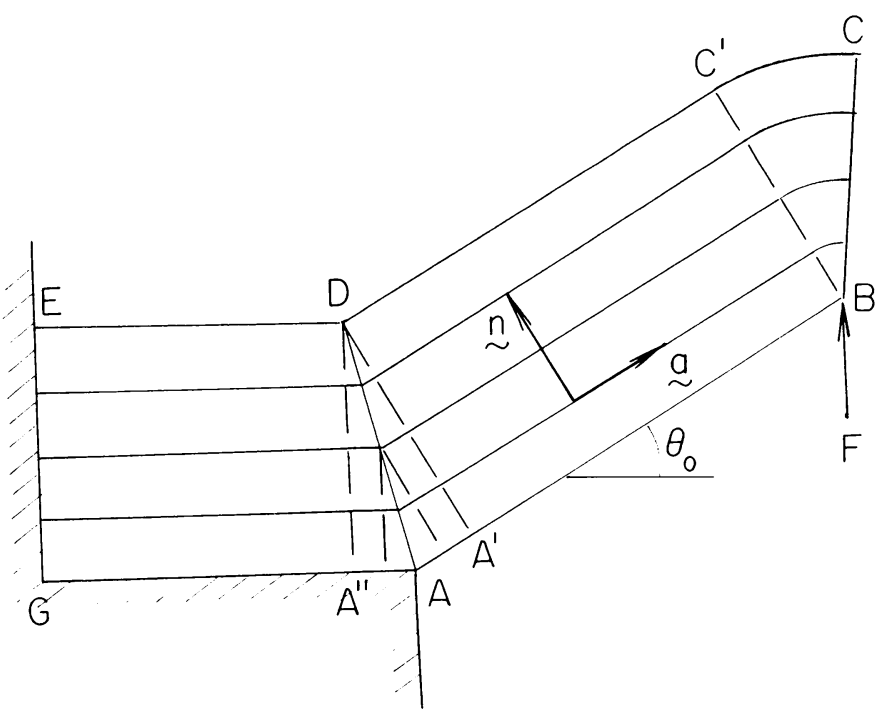

FIG. 3. Deformation with crimp line along $A D$.

general kinematical result $[2,3,6]$ that $k-\theta$ is constant along each fiber, except where the fiber crosses a crimp line. At a crimp line where the fiber angle changes discontinuously by the amount $\Delta \theta$, a simple geometrical construction shows that the amount of shear changes by the amount

$$
\Delta k=2 \tan (\Delta \theta / 2) .
$$

In both of the deformations considered here there is a simply sheared region in which the deflection angle is $\theta_{0}$ and the amount of shear is $k_{0}$, say. For the smooth deformation (Fig. 2) both $\theta$ and $k$ are zero in the region $X<0$, so the constancy of $k-\theta$ implies that

$$
k_{0}=\theta_{0} .
$$

In the deformation with a crimp line (Fig. 3), $\theta$ and $k$ are zero to the left of the crimp line, so $(2.1)$ shows that

$$
k_{0}=2 \tan \left(\theta_{0} / 2\right)
$$

Then for both deformations,

$$
k=\theta+k_{0}-\theta_{0} .
$$

For Fig. 2 this is valid everywhere, but for Fig. 3 it is valid only to the right of the crimp line $A D$.

In both solutions the fibers lie along concentric circular arcs in the region $B C C^{\prime}$, and the length of the $\operatorname{arc} C C^{\prime}$ is $H k_{0}$. It follows that on the end $B C$, the fiber angle is $\theta_{0}-k_{0}$, so the amount of shear is zero on the end for both deformations. This is compatible with the absence of shearing traction there.

Let $\mathrm{a}$ and $\mathrm{n}$ be unit vectors tangential and normal to the fibers, and let $\boldsymbol{\sigma}$ be the stress. When we speak of the shearing stress $S$, we mean the component a.d.n. For an elastic material, $S$ is a function $S(k)$ of the amount of shear. Since the amount of shear is constant in the region $A^{\prime} B C^{\prime} D$, the total shearing force on a normal cross-section in this region is 
$H S\left(k_{0}\right)$. This must be equal to the component of the end load $\mathrm{F}$ parallel to the crosssection. (This would imply the presence of a straight section if we had not taken it as given $[2,3]$.) This condition determines the deflection angle $\theta_{0}$ :

$$
H S\left(k_{0}\right)=F \cos \theta_{0},
$$

where $k_{0}$ is defined by (2.2) or (2.3). We assume that the solution $\theta_{0}$ is small enough that $H \theta_{0}$ is less than $L$ in Fig. 2 and $H k_{0} / 2$ is less than $L$ in Fig. 3; otherwise there is no simply sheared region $A^{\prime} B C^{\prime} D$ and the solution has a different form [3].

In addition to the two deformations considered here, there are other statically and kinematically admissible solutions in which the fiber angle changes from zero to $\theta_{0}$ partly through a crimp line and partly continuously. In Sec. 4 we exhibit the stress fields for the two deformations considered here; stress fields for the other solutions could be derived in the same way. Thus, under the usual kinematic and equilibrium conditions, the solution is highly non-unique.

3. Energy minimization. The non-uniqueness of the solution is removed by requiring the total energy of the body and its load to be a minimum, or at least stationary. Stationary energy implies equilibrium, but in the present theory the converse is not true.

Let $W(k)$ be the elastic strain energy per unit volume. This is related to the shearing stress by $S(k)=W^{\prime}(k)$. Let $E$ be the total potential energy of the deformed body, including the total strain energy and the energy $-F V$ of the load, $V$ being the vertical displacement of the load.

We recall that $k=\theta$ for the deformation in Fig. 2, and note that $\theta$ is constant along radial lines in the regions $D A A^{\prime}$ and $B C C^{\prime}$. In these regions, the total energy in a sector with vertex angle $d \theta$ is $W(\theta) H^{2} d \theta / 2$. With other results that are obvious by inspection, we find that the total energy for Fig. 2 is

$$
\begin{array}{rl}
E_{1}=\int_{0}^{k_{0}} & W(\theta) H^{2} d \theta+H\left(L-H \theta_{0}\right) W\left(\theta_{0}\right) \\
& \quad-F\left[H\left(1-\cos \theta_{0}\right)+\left(L-H \theta_{0}\right) \sin \theta_{0}\right] .
\end{array}
$$

Similarly, the total energy for the deformation in Fig. 3 is

$$
E_{2}=\frac{1}{2} \int_{0}^{\theta_{0}} W(k) H^{2} d k+H\left[L-\left(H k_{0} / 4\right)\right] W\left(k_{0}\right)-F L \sin \theta_{0} .
$$

The derivative of $E_{1}$ with respect to $\theta_{0}$ is

$$
\partial E_{1} / \partial \theta_{0}=\left(L-H \theta_{0}\right)\left[H S\left(\theta_{0}\right)-F \cos \theta_{0}\right] .
$$

Since we assume that $L$ exceeds $H \theta_{0}$, the variation (3.3) vanishes if and only if the equilibrium condition (2.5) is satisfied. At the value of $\theta_{0}$ determined by that condition, the second derivative of $E_{1}$ is

$$
\partial^{2} E_{1} / \partial \theta_{0}^{2}=\left(L-H \theta_{0}\right)\left[H S^{\prime}\left(\theta_{0}\right)+F \sin \theta_{0}\right] .
$$

This is positive and thus the energy is minimized, if $S(k)$ is an increasing function of $k$.

In contrast, $E_{2}$ is not even stationary at equilibrium. With (2.3), we find that

$$
\partial E_{2} / \partial \theta_{0}=(1 / 4) \sec ^{2}\left(\theta_{0} / 2\right)\left[H^{2} W_{0}+\left(4 L-H k_{0}\right) H S_{0}\right]-F L \cos \theta_{0},
$$

where $W_{0}=W\left(k_{0}\right)$ and $S_{0}=S\left(k_{0}\right)$. On eliminating $F$ by using $(2.5)$, we find that at the 
equilibrium value of $\theta_{0}$ the variation is

$$
\partial E_{2} / \partial \theta_{0}=(1 / 4) \sec ^{2}\left(\theta_{0} / 2\right)\left[H^{2}\left(W_{0}-k_{0} S_{0}\right)+4 H L S_{0} \sin ^{2}\left(\theta_{0} / 2\right)\right] .
$$

Since the equilibrium value of $\theta_{0}$ does not depend on $L$, the variation (3.6) can vanish for at most one value of $L$, purely by accident if at all.

Since the variation of $E_{2}$ with respect to $\theta_{0}$ is not zero at equilibrium, we reject the deformation in Fig. 3. In Sec. 5 we show that the crimp line in the solution is responsible for the non-stationary energy. All solutions with crimp lines can be rejected on the same basis.

4. Stress and equilibrium. In the present section we verify that the deformations in Figs. 2 and 3 are statically admissible.

For plane deformations of an ideal fiber-reinforced material, the stress has the form [1]

$$
\sigma=T \mathrm{aa}-P \mathrm{nn}+S(\mathrm{an}+\mathrm{na})+S_{33} \mathrm{kk},
$$

where $a$ and $n$ are unit vectors tangential and normal to the fibers in the plane of deformation and $\mathbf{k}$ is a unit vector normal to this plane. $S$ and $S_{33}$ are material response functions. $T$ and $P$ are reactions to the kinematic constraint conditions and are not related to the amount of shear by constitutive equations.

For a material with initially parallel fibers, the equilibrium equation $\nabla \cdot \sigma=0$ yields [1]

$$
\mathrm{a} \cdot \nabla T=2 S / r_{a}-\mathrm{n} \cdot \nabla S
$$

and

$$
\mathrm{n} \cdot \nabla P-P / r_{a}=T / r_{a}+\mathrm{a} \cdot \nabla S .
$$

Here $r_{a}$ is the signed radius of curvature of the fiber at the point considered, defined in terms of the fiber angle $\theta$ by

$$
l / r_{a}=\mathbf{a} \cdot \nabla \theta
$$

At a crimp line, let $\boldsymbol{\nu}$ and $\tau$ be unit vectors normal and tangential to the line (Fig. 4). Define

$$
\sigma_{\tau \nu}=\tau \cdot \sigma \nu \text { and } \sigma_{\nu \nu}=\nu \cdot \sigma \nu \text {. }
$$

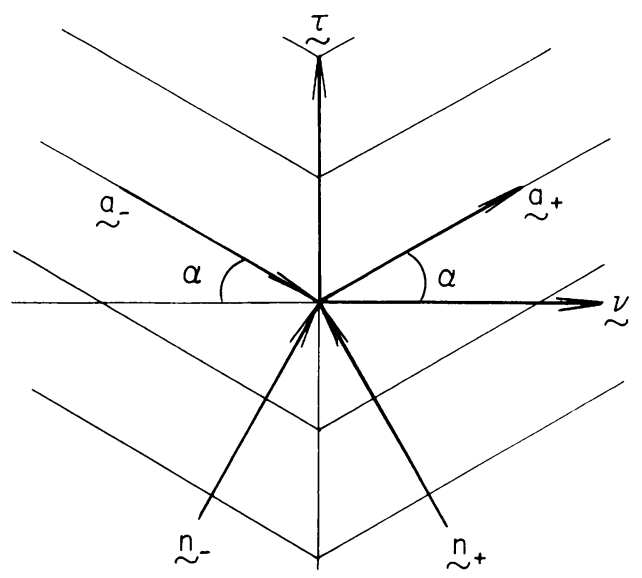

FIG. 4. Crimp line. 
With (4.1), these stress components are given to the right of the line in Fig. 4 by

$$
\sigma_{\tau \nu}=\frac{1}{2}(T+P) \sin 2 \alpha+S \cos 2 \alpha
$$

and

$$
\tau_{\nu v}=T \cos ^{2} \alpha-P \sin ^{2} \alpha-S \sin 2 \alpha .
$$

On the left side of the line, $\alpha$ is replaced by $-\alpha$ and $T, P$, and $S$ by their values on that side. Equilibrium requires that $\sigma_{\tau \nu}$ and $\sigma_{\nu \nu}$ be continuous across the line. From these continuity conditions, the values $T_{-}$and $P_{-}$on the left of the line can be found in terms of the values $T_{+}$and $P_{+}$on its right:

$$
T_{-}=T_{+} \cos 2 \alpha-P_{+} 2 \sin ^{2} \alpha-\Sigma S \sin 2 \alpha-\Delta S \cos 2 \alpha \tan \alpha
$$

and

$$
P_{-}=-T_{+} 2 \cos ^{2} \alpha-P_{+} \cos 2 \alpha+\Sigma S \sin 2 \alpha-\Delta S \cos 2 \alpha \operatorname{ctn} \alpha,
$$

where

$$
\Sigma S=S_{+}+S_{-} \quad \text { and } \quad \Delta S=S_{+}-S_{-} .
$$

Integration of (4.2) and (4.3) is easily carried out by methods that have been explained in detail elsewhere $[1,2,3,4]$. In the region $B C C^{\prime}$, for either deformation we obtain

$$
T=W[2-H \delta(Y-H)], \quad P=Y^{-1}\left[\left(S^{\prime}+2 W\right)(H-Y)-H W\right] .
$$

Here and in the formulas below, $X$ and $Y$ are the coordinates that the particle had when the body was undeformed. Thus $Y$ is constant along fibers. The term in $T$ involving a Dirac delta represents a finite force in the upper boundary fiber, which arises from the shearing stress discontinuity across that fiber. This surface tension causes a discontinuity in $P$, so that the value of $P$ at $Y=H$ in (4.11) is not the prescribed external value $P=0$.

In the simply sheared section of the slab, for both deformations $P=0$ and

$$
T=W_{0}[2-H \delta(Y-H)]+S_{0}\left(X-L+H k_{0}\right) \delta(Y-H)+\left[F_{0}-S_{0}(X-L)\right] \delta(Y) \text {. }
$$

where $W_{0}$ and $S_{0}$ are the constant values of $W$ and $S$ in this section. $F_{0}$ is the force in the lower boundary fiber just inside the point where the load is applied. This value is at first unknown because of the complicated singularity at the loaded point. It is found by using the global equilibrium condition that the resultant tension on the line $B C^{\prime}$ must have the value $F \sin \theta_{0}$. On that line, $X=L-H k_{0}$ on the top fiber and $X=L$ on the bottom one. Hence,

$$
F_{0}=F \sin \theta_{0}-H W_{0} .
$$

The preceding results are valid for both of the deformations under consideration, being different for the two deformations only because the variation of $k$ with position is not the same.

In the smooth deformation (Fig. 2), the reactions in the region $D A A^{\prime}$ are

$$
T=W[2-H \delta(Y)]+F_{1} \delta(Y)
$$

and

$$
P=(H-Y)^{-1}\left[\left(S^{\prime}+2 W\right) Y-H W+F_{1}\right],
$$


where

$$
F_{1}=S_{0}\left(L-H \theta_{0}\right)+F \sin \theta_{0}
$$

In the region $A D E G$,

$$
T=F_{1}[\delta(Y)-\delta(Y-H)] \text { and } P=-F \delta(X) .
$$

To obtain $T$ in (4.17) we have assumed that there is no shearing stress discontinuity across the fiber $A G$, so that the force in it stays at a constant value, the value $F_{1}$ given by (4.14) at the point $A$. The forces in the upper boundary fiber and the singular normal line $A D$ are found from global equilibrium considerations, because of the singularity at $D$.

For the deformation with a crimp (Fig. 3), on the right-hand side of the crimpline $P$ is zero and $T$ is given by (4.12), with $X=-H k_{0} / 2$ on the top fiber and $X=0$ on the bottom one:

$$
T=2 W_{0}-F_{2} \delta(Y-H)+F_{3} \delta(Y) \text {. }
$$

Here

$$
F_{2}=H W_{0}+S_{0}\left[L-\left(H k_{0} / 2\right)\right] \text { and } F_{3}=F_{0}+L S_{0} .
$$

The values of $T$ and $P$ on the left side of $A D$ are found by using (4.8) and (4.9), with $2 \alpha=$ $\theta_{0}$. Then $T$ is given throughout $A D E G$ by

$$
\begin{aligned}
T=2 W_{0} \cos \theta_{0}-S_{0}\left[\sin \theta_{0}+\cos \theta_{0} \tan \left(\theta_{0} / 2\right)\right] & \\
& +\cos \theta_{0}\left[F_{3} \delta(Y)-F_{2} \delta(Y-H)\right] .
\end{aligned}
$$

$P$ is determined in $D A A^{\prime \prime}$ by its values on $A D$ :

$$
\begin{aligned}
P=-4 W_{0} \cos ^{2}\left(\theta_{0} / 2\right)+S_{0}\left[\sin \theta_{0}-\cos \theta_{0}\right. & \left.\operatorname{ctn}\left(\theta_{0} / 2\right)\right] \\
& +\sin \theta_{0}\left[F_{2} \delta\left(X+H k_{0} / 2\right)-F_{3} \delta(X)\right] .
\end{aligned}
$$

The coefficients of the Dirac deltas in (4.21) are found most easily by considering the equilibrium of the points $A$ and $D$ directly. In $A^{\prime \prime} D E G, P$ is zero.

5. Energy variations at a crimp line. In a virtual change $d \theta_{0}$ of the deflection angle in Fig. 3, the crimp line $A D$ pivots through an angle $d \theta_{0} / 2$ and thus passes over some material elements. This material is displaced only infinitesimally, but the displacement gradient in it changes by a finite amount. The usual proof that stationary energy is equivalent to equilibrium is not valid in such cases, because the usual proof assumes that a small variation in displacement causes only a small variation in the displacement gradient.

We may regard the crimp line as stationary and view material as moving across the line during a virtual deformation. In a movement from the minus side to the plus side in Fig. 4, the change of stored energy per unit volume is

$$
\Delta W=W\left(k_{+}\right)-W\left(k_{-}\right) .
$$

The work per unit volume done on an element crossing the line is $\sigma_{\tau \nu} \Delta k$. The quantity

$$
\phi=\Delta W-\sigma_{\tau \nu} \Delta k
$$

represents the amount of energy created, per unit volume. This is zero when $\Delta k$ is infinitesimal, but generally not zero when there is a finite discontinuity. Although a negative value of $\phi$ is physically admissible as energy dissipation, the opposite virtual 
deformation would then require energy creation. Thus crimp lines are physically inadmissible in problems in which they can move during a virtual deformation. They are admissible in problems in which they are imposed by displacement boundary conditions, because in such problems they cannot move in any kinematically admissible virtual displacement.

As a check, we now verify directly that the non-zero value (3.6) of the energy variation for the deformation in Fig. 3 is due to energy changes in material crossing the crimp line. Let $r$ be distance along the line $A D$, measured from the point $A$. A segment of length $d r$ on this line sweeps out an area $d r\left(r d \theta_{0} / 2\right)$ when the deflection angle increases by the amount $d \theta_{0}$. Let $\Phi d \theta_{0}$ be the total energy production for the whole line. Then, with $r=Y \mathrm{sec}$ $\left(\theta_{0} / 2\right)$

$$
\Phi=\frac{1}{2} \sec ^{2}\left(\theta_{0} / 2\right) \int_{0}^{H} \phi Y d Y .
$$

To evaluate this we first evaluate $\sigma_{\tau \nu}$. Since $P=0$ on the righthand side of $A D$ and $T$ is given there by (4.18), from (4.6) we obtain

$$
\sigma_{\tau \nu}=\frac{1}{2} \sin \theta_{0}\left[2 W_{0}-F_{2} \delta(Y-H)+F_{3} \delta(Y)\right]+S_{0} \cos \theta_{0} .
$$

Then

$$
\int_{0}^{H} \sigma_{\tau \nu} Y d Y=\frac{1}{2} H^{2}\left(W_{0} \sin \theta_{0}+S_{0} \cos \theta_{0}\right)-\frac{1}{2} H F_{2} \sin \theta_{0} .
$$

In (5.2), $\Delta W=W_{0}$ and $\Delta k=k_{0}$. By combining these results in (5.3), we obtain

$$
2 \Phi \cos ^{2}\left(\theta_{0} / 2\right)=\frac{1}{2} H^{2} W_{0}-\frac{1}{2} H^{2} k_{0}\left(W_{0} \sin \theta_{0}+S_{0} \cos \theta_{0}\right)+\frac{1}{2} H F_{2} k_{0} \sin \theta_{0} .
$$

Then by using the value of $k_{0}$ given by (2.3) and the value of $F_{2}$ given by (4.19), we find that $\Phi$ is exactly equal to the value of $d E_{2} / d \theta_{0}$ given by (3.6).

6. Energy dissipation in peeling. With reference to the correct solution (Fig. 2), we now consider a different matter. Let us suppose that the slab peels loose from the rigid foundation on a segment of length $d L$ at point $A$. The new deformation differs from that found previously only in that the length $L$ of the unsupported section is increased by the amount $d L$. Let $\mathcal{G} d L$ be the energy dissipated during the extra deformation. From (3.1) we find that the energy release rate is

$$
\mathcal{G}=-\partial E_{1} / \partial L=F \sin \theta_{0}-H W\left(\theta_{0}\right) .
$$

By using (2.5) to eliminate $F$ and then rearranging the resulting expression, we find that

$$
\mathcal{G}=H \sin \theta_{0} \tan \theta_{0}\left(d / d \theta_{0}\right)\left[W\left(\theta_{0}\right) / \sin \theta_{0}\right] .
$$

This form of the result shows that the energy release rate is positive, under the mild assumption that $W(k) / \sin k$ is an increasing function of $k$.

The energy dissipation takes place at the point $D$. This can be verified by taking a small material element including $D$ as a free body and computing the energy release rate for this element. The stress field needed in the computation is given by (4.14) and (4.15). The displacement field for the extra deformation is $\mathrm{u}=(\mathrm{a}-\mathrm{i}) d L$, where $\mathrm{i}$ is a unit vector in the $x$-direction. We have carried out such a computation and have verified that it yields the value $(6.1)$.

In the linearized, infinitesimal-deformation theory version of the present problem, the 
energy release rate is easily found to be $F^{2} / 2 G H$ [7], and this agrees with the limit of (6.1) when (2.5) is taken into account. However, the energy dissipation in the infinitesimal theory is distributed along the line $A D$. Rogers [5] has commented on the differences between the finite and infinitesimal solutions in a similar problem.

In elastic fracture mechanics [8] the energy release rate in crack advance can be calculated from conditions at the tip of the crack, and indeed the energy release rate for the whole body is the same as that for an arbitrarily small neighborhood of the crack tip. This suggests that if the present problem were solved for an elastic material with small but finite extensibility and compressibility, the singularity at $D$ would disappear and be replaced by a singularity at $A$. On the other hand, it is possible that an elastic solution might exhibit a crease at $D$ that could contribute to energy dissipation. This could not occur in an infinitesimal-deformation theory because creasing is essentially a finite deformation.

Because the result (6.1) was obtained by global methods, we think it is likely that it is an adequate approximation to the energy release rate for real fiber-reinforced materials. However, the locale of this energy dissipation is in doubt. In contrast to normal expectations, the present results suggest that the energy dissipation in a real material will not be entirely at the crack tip.

\section{REFERENCES}

[1] A. C. Pipkin and T. G. Rogers, Plane deformations of incompressible fiber-reinforced materials, J. Appl. Mech. 38, 634-40 (1971)

[2] A. C. Pipkin, Finite deformations of ideal fiber-reinforced composites, in Composite materials, Vol. 2: Mechanics of composite materials, ed. G. P. Sendeckyj, Academic Press, New York, 1974, pp. 251-308

[3] B. Kao and A. C. Pipkin, Finite buckling of fiber-reinforced columns, Acta Mech. 13, 265-80 (1972)

[4] G. C. Everstine and T. G. Rogers, A theory of machining of fiber-reinforced materials, J. Comp. Mat. 5, 94-106 (1971)

[5] T. G. Rogers, Finite deformations of strongly anisotropic materials, in Theoretical rheology, ed. Hutton, Pearson, \& Walters, Wiley, New York, 1975, pp. 141-168

[6] T. G. Rogers and A. C. Pipkin, Finite lateral compression of a fibre-reinforced tube, QJMAM 24, 311-30 (1971)

[7] V. Sanchez-M. and A. C. Pipkin, Energy release rate for cracks in ideal composites, Int. J. Solids Str. 13, $571-8(1977)$

[8] Rice, J. R., Mathematical analysis in the mechanics of fracture, in Fracture. Vol. II, Academic Press, New York, 1968, pp. 191-311 